\title{
Impact of upper airway configuration assessed by CT on CPAP titration in OSA patients during Müller's maneuver
}

\section{Qichang Lin ( $\square$ chang4e@126.com )}

First Affiliated Hospital of Fujian Medical University

\section{Han-Sheng Xie}

First Affiliated Hospital of Fujian Medical University

\section{Jie-feng Huang}

First Affiliated Hospital of Fujian Medical University

Jian-Ming Zhao

First Affiliated Hospital of Fujian Medical University

Ai-Ming Zeng

First Affiliated Hospital of Fujian Medical University

\section{Bi-Ying Wang}

First Affiliated Hospital of Fujian Medical University

\section{Gong-Ping Chen}

First Affiliated Hospital of Fujian Medical University

\section{Research article}

Keywords: Obstructive sleep apnea, Upper airway configuration, CPAP titration level, Müller's maneuver, Computed tomography

Posted Date: July 10th, 2019

DOI: https://doi.org/10.21203/rs.2.11197/v1

License: (a) (1) This work is licensed under a Creative Commons Attribution 4.0 International License. Read Full License 


\section{Abstract}

Objectives Continuous positive airway pressure (CPAP) is the current gold-standard treatment for moderate to severe obstructive sleep apnea (OSA), and upper airway anatomy plays an increasingly important role in evaluating the efficacy of CPAP therapy. The aim of this observational study was to investigate the influence of upper airway anatomy on CPAP titration in OSA patients assessed by computed tomography (CT) during Müller's maneuver. Methods Consecutive patients under investigation for OSA by undergoing polysomnography and CT scan of the upper airway while awake were enrolled. Successful full-night manual titration was performed to determine the optimal CPAP pressure level for OSA patients in supine position using a nasal mask. Results A total of 157 subjects ( 134 males and 23 females) were included. Both apnea-hypopnea index (AHI) and LaSO2 significantly correlated with CPAP titration level, upper airway length (UAL), distance from mandibular plane to hyoid bone (MPH), and neck circumference (all $p<0.05)$. There were significant positive correlations between CPAP titration level and $\operatorname{UAL}(r=0.348, p=0.000)$ and MPH $(r=0.313, p=0.002)$. Stepwise multiple linear regression analyses were performed to evaluate the independent predictors of AHI, LaSO2, and CPAP titration level. CPAP titration level was identified as an independent explanatory variable for $\mathrm{AHI}$ and LaSO2 after adjustment for confounders. Multiple linear regression analyses also indicated that body mass index (BMI) and UAL were independently associated with CPAP titration level (all $p<0.05$ ). Conclusions Upper airway abnormalities combined with anthropometric parameters play important roles in CPAP titration for OSA patients, providing additional insight into the factors influencing OSA treatment strategies. UAL and BMI should be taken into consideration when choosing CPAP titration level to improve CPAP compliance.

\section{Background}

Obstructive sleep apnea (OSA) is characterized by repetitive occlusion of the upper airway during sleep as a result of anatomic and physiologic abnormalities, which results in partial or complete airflow obstruction. Continuous positive airway pressure (CPAP), the primary therapy for patients with OSA, reduces its cardiovascular morbidity including systemic hypertension, left ventricular systolic function, and sympathetic nervous activity [1]. Despite its proven efficacy, a variety of factors may contribute to poor CPAP compliance, such as technical problems with the device, individual patient characteristics, and psychosocial issues [2]. The titration level of CPAP treatment appears to be an important influential factor in CPAP compliance, given that upper airway narrowing was improved and airway resistance was decreased after surgical operation and CPAP lately has been compliance [3-5].

Obstruction in any upper airway can interfere with CPAP titration levels because of increasing negative pressure in the pharyngeal airway and the predisposition of the pharynx to collapse, which may contribute to the underlying pathogenesis of poor CPAP compliance. Therefore, characterizing the mechanisms leading to upper airway collapse in OSA patients could pave the way to guide treatment options. Our previous study of the important pathogenic role played by upper airway abnormalities in OSA showed that assessment by computed tomography (CT) imaging during Müller's maneuver offers great advantages in detecting sites of upper airway obstruction [6]. However, to the best of our knowledge 
there are no studies that focus on the predictive value of CT imaging during Müller's maneuver with regard to CPAP titration levels.

For this reason we embarked on a study to evaluate in OSA patients the correlations between CPAP pressure and combined polysomnographic and structural changes in the upper airway by employing CT during Müller's maneuver. Our aim was to determine the optimal level of CPAP titration and find the relevant anatomic abnormalities that may influence CPAP compliance, thus enabling patients to better tolerate CPAP therapy.

\section{Methods}

\subsection{Subjects}

Individuals with a snoring problem who were referred to our sleep center with clinical suspicion of OSA were enrolled in our study from July 2013 to February 2015. All subjects were required to undergo full-night polysomnography (PSG). Exclusion criteria were as follows: (1) patients with nasal, oral, pharyngeal, or mandibular diseases; (2) previous CPAP treatment, use of oral appliance, or upper airway surgery; (3) other diseases including apparent respiratory, cardiac, or renal disease, and hypothyroidism. All patients were scheduled to undergo an assessment of the upper airway through CT scanning during Müller's maneuver (described below). Before the study, informed consent was obtained from all subjects and the local institutional review board approved the study protocol.

\subsection{Medical history and anthropometric measurements}

Demographic data including sex, age, and body mass index (BMI), as well as a detailed questionnaire on sleep symptoms, Epworth sleepiness scale, and history of alcohol consumption and smoking were collected from all subjects. Body weight and height were measured without shoes while lightly clothed in the morning, and BMI was calculated as weight $(\mathrm{kg}) /$ height $^{2}(\mathrm{~m})$. Waist circumference was measured around the middle between the 12th rib and the iliac crest, and neck circumference at the level of the laryngeal prominence, using a measuring tape.

\subsection{Polysomnography and CPAP titration}

Overnight PSG (P Series Sleep System; Compumedics; Melbourne, Australia) was performed. The parameters recorded included electroencephalography, electrooculography, electromyography, airflow by nasal and oral thermistors, respiratory effort by thoracic and abdominal impedance belts, arterial oxyhemoglobin saturation by pulse oximetry, snoring by tracheal microphone, and change of body position during sleep by a sensor. PSG recordings were manually scored for sleep stages by a physician according to the criteria of the American Academy of Sleep Medicine published in 2012. The apneahypopnea index (AHI) was defined as the number of apneic and hypopneic episodes per hour of polysomnographically recorded sleep time, and the oxygen desaturation index (ODI) was defined as the number of dips in oxygen saturation $\left(\mathrm{SpO}_{2}\right) 4 \%$ or greater per hour of polysomnographically recorded 
sleep time. Other polysomnographic parameters included the number of apneic episodes per hour of polysomnographically recorded sleep time (Al), the percentage of total sleep time spent with $\mathrm{SpO}_{2}<90 \%$ (T90\%), and lowest $\mathrm{O}_{2}$ saturation $\left(\mathrm{LaSO}_{2}\right)$.

Patients whose AHI was $\geq 15.0$ per hour after undergoing full-night PSG received automatic CPAP titration. When the average usage time of CPAP treatment was more than $4.0 \mathrm{~h}$ per night and the AHI decreased to $<5$ per hour, this was defined as good compliance, and the optimal level of CPAP titration was defined as the lowest effective pressure. The data were collected and analyzed electronically by a smartcard embedded in the CPAP devices.

\subsection{CT evaluation}

CT scanning was performed to measure the following parameters in all patients while under Müller's maneuver: anteroposterior (AP) and transverse (T) diameters at the level of the nasopharynx (NP) (upper limit: cranial base; lower limit: tip of uvula), oropharynx (OP) (upper limit: tip of uvula; lower limit: tip of the epiglottis), and hypopharynx (HP) (upper limit: at the level of vallecula; lower limit: at the level of cricoid cartilage). All the patients remained awake in the supine position with the Frankfort plane perpendicular to the floor. Slices in the axial plane extending from the skull base to the hypopharynx, below the level of the cricoid cartilage, were collected at 5-mm intervals, with a total scan time of $10 \mathrm{~s}$. A lateral scout view was firstly taken to determine and standardize the level of the scans during quiet tidal breathing. The minimal cross-sectional area within the NP, OP, and HP regions (mCSA-NP, mCSA-OP, and mCSA-HP) was obtained through measuring AP and $\mathrm{T}$, and the shape at each mCSA level was expressed as the ratio of AP to T (AP/T-NP, AP/T-OP, and AP/T-HP) during Müller's maneuver. Upper airway length (UAL) was defined as the vertical distance from the hard palate to the hyoid in the mid-sagittal plane. We also calculated the distance from the mandibular plane to hyoid bone (MPH). UAL and MPH were obtained from the lateral scout view during quiet respiration within the first $5 \mathrm{~s}$ (Fig. 1a). We did not recognize any problematic images (e.g., not in the neutral anatomic position while reviewing the CT scan). To avoid bias, every subject was taught several times how to perform Müller's maneuver until they were able to perform the standard maneuver. All measurements were done manually by one clinician who was blinded to the PSG data.

\subsection{Statistical analysis}

SPSS v.17.0 for Windows (SPSS, Chicago, IL) was used to analyze all data. Continuous variables were tested for normal distribution prior to subsequent statistical analysis. Data were presented as mean \pm standard deviation, median (interquartile range), and number (percentage) for normally distributed, skewed, and categorical data, respectively. Correlations between PSG variables and anthropometric parameters and CPAP titration level were assessed by the Pearson correlation test. Stepwise logistic regression analyses were performed to determine the independent predictors of $\mathrm{AHI}, \mathrm{LaSO}_{2}$, and $\mathrm{CPAP}$ titration level. Differences were considered significant when the $p$ value was less than 0.05 . 


\section{Results}

A total of 157 patients (134 men and 23 women) were enrolled in the study. The mean age was $46.40 \pm 11.72$ years, with mean BMI of $26.98 \pm 3.38 \mathrm{~kg} / \mathrm{m}^{2}$. Regarding the severity of OSA, the mean AHI was $46.42 \pm 22.28$ events/h and mean $\mathrm{LaSO}_{2} 69.98 \% \pm 14.24 \%$, ranging from $30 \%$ to $90 \%$. The mean CPAP titration level was $9.68 \pm 2.50 \mathrm{mmHg}$. The other parameters including anthropometric variables, measurements of upper airway by CT scan, and PSG variables are shown in Table 1.

Table 2 shows the correlations between the severity of OSA, CPAP titration level, and other variables. Both $\mathrm{AHI}$ and $\mathrm{LaSO}_{2}$ significantly correlated with CPAP pressure, UAL, MPH, and neck circumference (all $p<0.05)$. Significant positive correlations were found between the CPAP titration level and anthropometric variables, including age, neck circumference, and waist circumference. Moreover, Spearman correlation coefficient analysis showed significant positive correlations between the CPAP titration level and both UAL $(r=0.348, p=0.000)$ and MPH $(r=0.313, p=0.002)$.

Stepwise multiple linear regression analyses were performed to evaluate the independent predictors of $\mathrm{AHI}, \mathrm{LaSO}_{2}$, and CPAP titration level. CPAP titration level was identified as an independent explanatory variable for $\mathrm{AHI}$ and $\mathrm{LaSO}_{2}$ after adjustment for confounders. Furthermore, multiple linear regression analyses indicated that BMI and UAL were independently associated with CPAP titration level $(\beta=0.343$ and 0.275 , and $p=0.000$ and 0.000 , respectively) (Table 3 ).

\section{Discussion}

In this cross-sectional study, we found that the CPAP titration level partly explained the severity of OSA, and $\mathrm{BMI}$ and UAL were associated with CPAP titration level independent of a variety of relevant factors including age, neck circumference, waist circumference, MPH, mCSA-NP, mCSA-OP, and mCSA-HP, which suggested that $\mathrm{BMI}$ and UAL might be predictive factors for upper airway pressure.

Anatomic abnormalities and compliance of the upper airway constitute the main causes of OSA, by changing the size or caliber of the upper airway during sleep. Various methods including physical examination, flexible fiberoptic laryngoscopy, and imaging techniques have been used to assess the obstruction and collapse of the upper airway in OSA patients [7-9]. Our previous study of 358 snoring patients also found that NP narrowing, UAL, and MPH seem to play important physiopathogenic roles in OSA [6]. Some studies have reported that any obstruction of the upper airway would have an effect on CPAP titration level by interfering with the airflow [10-12], with a consequential impact on CPAP compliance.

Previous research evaluating the possible influential factors in CPAP titration, aimed at lowering economic costs and significantly increasing the tolerance of CPAP therapy, have produced varying results $[10,13,14]$. A systematic review by Camacho et al. [10] showed that BMI and ODI weighed most heavily in the equation that predicted the CPAP titration level. Bosi et al. [11] reported that PSG variables and 
anthropometric parameters had no significant independent predictive value for CPAP titration, with the exception of BMI. A study enrolling severe OSA and moderately obese patients analyzed the predictors for CPAP therapy pressure among PSG variables, anthropometric parameters, and cephalometric measurements, and found that cephalometric measurements combined with PSG variables and BMI could predict more accurately the CPAP therapy pressure [14]. Other investigators reported that subjects with higher BMI lowered CPAP adherence, citing weight loss as an effective means to improve OSA treatment outcomes [15]. It has also been proposed that obesity-induced hypoventilation might account for poor adherence to CPAP therapy in morbidly obese patients with OSA [16, 17]. Similar to previous research, in the present study we also found a significant association between the CPAP titration level and BMI.

It is noteworthy that our study not only involved PSG variables, anthropometric parameters, and cephalometric or endoscopic assessment under Müller's maneuver to predict the CPAP titration level, but also focused on the role of CT evaluation as a predictor of the CPAP titration level. Indeed we found that UAL, defined as the vertical distance from the hard palate to the hyoid in the mid-sagittal plane, was associated with the level of CPAP titration. It has been suggested that UAL plays an important role in the assessment of pharyngeal collapse, whereby reduced UAL would improve the severity of OSA [18-20]. The relationship between UAL and the collapsibility of the pharynx could be interpreted by Bernoulli's law, which states that an inviscid fluid flowing through a tube is susceptible to an increase in velocity and a decrease in pressure [21]. Because the pharynx lacks rigid bony structures, it is more prone to collapse when UAL is longer, based on the rules of buckling of cylindrical shells [22]. Therefore, longer UAL might become a possible mechanism in explaining the increased collapsibility of the upper airway in patients with OSA, which would further increase the CPAP titration level. Ben Ner et al. [23] found in their investigation of 108 male OSA patients that UAL, which indicates pharynx length, correlated significantly with OSA severity, while BMI and UAL had an additive impact on OSA. Our study also demonstrated that both $\mathrm{AHI}$ and $\mathrm{LaSO}_{2}$ significantly correlated with UAL and MPH. Furthermore, BMI and UAL were independently associated with CPAP titration level, which plays an important role in CPAP compliance.

The present study clearly has some limitations. First, the subjects enrolled carried a clinical suspicion of OSA and were not drawn from the general population, which might lead to selection bias. Second, CT scanning puts patients at risk for radiation exposure compared with other clinical diagnostic methods in the diagnosis of OSA. Nevertheless, CT scanning is noninvasive and provides multiple-level, rapidsequence scans. Third, the CT scan was conducted on patients when they were awake, while OSA is a dynamic phenomenon that occurs during sleep. However, given the impracticality of performing CT on sleeping patients, we evaluated the upper airways while under Müller's maneuver, which adequately simulates upper airway collapse during sleep.

In conclusion, we found that upper airway abnormalities combined with anthropometric parameters are more likely to play an important role in CPAP titration in OSA patients, and multiple logistic regression analysis provided additional insight into the factors affecting OSA treatment. Our study suggests that 
UAL and BMI should be taken into consideration in improving CPAP compliance when choosing additional therapies such as an oral appliance or surgery.

\section{Abbreviations}

CPAP Continuous positive airway pressure

OSA obstructive sleep apnea

CT computed tomography

UAL upper airway length

MPH distance from mandibular plane to hyoid bone

PSG polysomnography

AHI hypopnea index

Al the number of apneic episodes per hour of polysomnographically recorded sleep time

BMI body mass index

ODI oxygen desaturation index

$\mathrm{SpO}_{2}$ oxygen saturation

$\mathrm{LaSO}_{2}$ lowest $\mathrm{O} 2$ saturation

T90\% the percentage of total sleep time spent with $\mathrm{SpO}_{2}<90 \%$

mCSA-NP the minimal cross-sectional area within the nasopharynx

mCSA-OP he minimal cross-sectional area within the oropharynx

mCSA-HP the minimal cross-sectional area within the hypopharynx

\section{Declarations}

Ethics approval and consent to participate: This research was approved by institutional review board in the First Affiliated Hospital of Fujian Medical University.All patients gave their informed consents.

Consent for publication: Not applicable

Availability of data and material: The datasets used and analysed during the current study are available from the corresponding author on reasonable request 
Competing interests: The authors declare that they have no competing interests

Funding: no funding

Authors' contributions: Han-Sheng Xie sequence/data analysis and preparation of the manuscript; Jiefeng Huang,, study design and preparation of the manuscript; Bi-Ying Wang, study design, experiments and manuscript preparation; Qi-Chang Lin, contributed in experimental design; Gong-Ping Chen, experiments;Ai-Ming Zeng, analyzed data; Jian-Ming Zhao, collected data; Han-Sheng Xie, Jie-feng Huang and Jian-Ming Zhao contributed equally to this work. All authors read and approved the final manuscript

\section{Acknowledgements:}

We thank Hugh McGonigle, from Liwen Bianji, Edanz Group China (www.liwenbianji.cn/ac), for editing the English text of a draft of the manuscript.

\section{References}

1. Vanderveken, O. M. et al. Cardiovascular implications in the treatment of obstructive sleep apnea. $J$ Cardiovasc Transl Res 4, 53-60 (2011).

2. Garvey, J. F. \& McNicholas, W. T. Continuous positive airway pressure therapy: new generations. Indian J. Med. Res. 131, 259-266 (2010).

3. Friedman, M., Soans, R., Joseph, N., Kakodkar, S. \& Friedman, J. The effect of multilevel upper airway surgery on continuous positive airway pressure therapy in obstructive sleep apnea/hypopnea syndrome. Laryngoscope 119, 193-196 (2009).

4. Hong, S. D. et al. Effect of uvulopalatopharyngoplasty on CPAP compliance. Eur Arch Otorhinolaryngol 272, 1437-1442 (2015).

5. Azbay, S., Bostanci, A., Aysun, Y. \& Turhan, M. The influence of multilevel upper airway surgery on CPAP tolerance in non-responders to obstructive sleep apnea surgery. Eur Arch Otorhinolaryngo/ 273, 2813-2818 (2016).

6. Huang, J.-F. et al. Assessment of Upper-Airway Configuration in Obstructive Sleep Apnea Syndrome With Computed Tomography Imaging During Müller Maneuver. Respir Care 61, 1651-1658 (2016).

7. Stuck, B. A. \& Maurer, J. T. Airway evaluation in obstructive sleep apnea. Sleep Med Rev 12, 411-436 (2008).

8. Tejan, J., Medina, M. \& Ulualp, S. O. Comparative Assessment of Drug-Induced Sleep Endoscopy Scoring Systems in Pediatric Sleep Apnea. Laryngoscope 16, 504 (2019).

9. Liu, S. Y.-C. et al. Static craniofacial measurements and dynamic airway collapse patterns associated with severe obstructive sleep apnoea: a sleep MRI study. Clin Otolaryngol 41, 700-706 (2016).

10. Camacho, M., Riaz, M., Tahoori, A., Certal, V. \& Kushida, C. A. Mathematical Equations to Predict Positive Airway Pressures for Obstructive Sleep Apnea: A Systematic Review. Sleep Disord 2015, 293868-11 (2015). 
11. Bosi, M., De Vito, A., Vicini, C. \& Poletti, V. The predictive value of Muller's maneuvre for CPAP titration in OSAHS patients. Eur Arch Otorhinolaryngol 270, 2345-2351 (2013).

12. Lan, M.-C. et al. The predictive value of drug-induced sleep endoscopy for CPAP titration in OSA patients. Sleep Breath 22, 949-954 (2018).

13. Lai, C.-C. et al. Clinical predictors of effective continuous positive airway pressure in patients with obstructive sleep apnea/hypopnea syndrome. Laryngoscope 125, 1983-1987 (2015).

14. Akahoshi, T. et al. Predicting optimal continuous positive airway pressure in Japanese patients with obstructive sleep apnoea syndrome. Respirology 14, 245-250 (2009).

15. Park, P. et al. Influencing factors on CPAP adherence and anatomic characteristics of upper airway in OSA subjects. Medicine (Baltimore) 96, e8818 (2017).

16. Schäfer, H., Ewig, S., Hasper, E. \& Lüderitz, B. Failure of CPAP therapy in obstructive sleep apnoea syndrome: predictive factors and treatment with bilevel-positive airway pressure. Respir Med $\mathbf{9 2}$, 208-215 (1998).

17. Yao, Q. et al. Localizing Effects of Leptin on Upper Airway and Respiratory Control during Sleep. Sleep 39, 1097-1106 (2016).

18. Segal, Y., Malhotra, A. \& Pillar, G. Upper airway length may be associated with the severity of obstructive sleep apnea syndrome. Sleep Breath 12,311-316 (2008).

19. Kim, E. J. et al. Upper airway changes in severe obstructive sleep apnea: upper airway length and volumetric analyses using 3D MDCT. Acta Otolaryngol. 131, 527-532 (2011).

20. Sutherland, K. et al. Effect of weight loss on upper airway size and facial fat in men with obstructive sleep apnoea. Thorax 66, 797-803 (2011).

21. Fajdiga, I. Snoring imaging: could Bernoulli explain it all? Chest 128, 896-901 (2005).

22. Ekstrom RE (1963) Buckling of cylindrical shells under combined torsion and hydrostatic pressure. Exp Mech 3:192-197.

23. Ben Ner, D., Carmel-Neiderman, N. N., Fliss, D. M., Haas, N. \& Rosenzweig, E. The Interaction Between Craniofacial Computed Tomographic Dimensional Parameters and BMI in Obstructive Sleep Apnea. J Maxillofac Oral Surg 18, 299-306 (2019).

\section{Tables}


Table 1 Anthropometric variables, measurements of upper airway by CT scan and PSG variables

\begin{tabular}{|c|c|c|}
\hline Variables & Mean \pm SD & Range \\
\hline Males (n, \%) & $157(87.22 \%)$ & - \\
\hline Females (n, \%) & 23(12.78\%) & - \\
\hline Age, years? & $46.40 \pm 11.72$ & $21.00-78.00$ \\
\hline BMI, $\mathrm{kg} / \mathrm{m}^{2} \square$ & $26.98 \pm 3.38$ & $20.08-37.20$ \\
\hline AHI, events/h & $46.42 \pm 22.28$ & $15.00-96.20$ \\
\hline AI, events/h & $40.33 \pm 24.18$ & $13.00-91.70$ \\
\hline ODI, events/h & $38.18 \pm 23.87$ & $0.50-93.60$ \\
\hline $\mathrm{LaSO}_{2}, \%$ & $69.98 \pm 14.24$ & $31.00-90.00$ \\
\hline CPAP titration level & $9.68 \pm 2.50$ & $3.20-18.80$ \\
\hline Neck circumference, cm & $39.25 \pm 3.50$ & $30.00-53.00$ \\
\hline Waist circumference, cm & $95.27 \pm 9.24$ & $65.00-128.00$ \\
\hline mCSA-NP & $0.90 \pm 0.93$ & $0.00-8.10$ \\
\hline mCSA-OP & $2.63 \pm 1.66$ & $0.00-9.38$ \\
\hline mCSA-HP & $1.35 \pm 1.31$ & $0.00-5.33$ \\
\hline UAL & $7.65 \pm 1.04$ & $1.00-9.87$ \\
\hline $\mathrm{MPH}$ & $2.21 \pm 0.83$ & $0.45-4.29$ \\
\hline
\end{tabular}

OSA obstructive sleep apnea, CPAP continuous positive airway pressure, BMI Body mass index , AHI apneahypopnea index, ODI oxygen desaturation index, T90\% the percentage of total sleep time spent with SpO2<90 $\%$, LaSO2 lowest O2 saturation,mCSA-NP, mCSA-OP, mCSA-HP: The minimal cross sectional area within the nasopharynx, oropharynx and hypopharynx regions; AP/T anteroposterior/transverse axis ratio; UAL upper airway length; MPH the distance from mandibular plane to hyoid bone. 
Table 2 Correlations between between the severity of OSA, CPAP titration level and the other variables

AHI, $r(p) \quad$ CPAP titration level, $r(p)$

\begin{tabular}{llll}
\hline Age & $-0.150\left(0.045^{*}\right)$ & $-0.080(0.290)$ & $0.188\left(0.012^{*}\right)$ \\
Neck circumference & $0.161\left(0.032^{*}\right)$ & $-0.289\left(0.000^{*}\right)$ & $0.289\left(0.000^{*}\right)$ \\
Waist circumference & $0.096(0.201)$ & $-0.304\left(0.000^{*}\right)$ & $0.275\left(0.000^{*}\right)$ \\
& & & \\
\hline BMI & $0.053(0.479)$ & $-0.100(0.184)$ & $0.139(0.063)$ \\
\hline AHI & - & $-0.494\left(0.000^{*}\right)$ & $0.443\left(0.000^{*}\right)$ \\
\hline ODI & $0.860\left(0.000^{*}\right)$ & $-0.737\left(0.000^{*}\right)$ & $0.376\left(0.000^{*}\right)$ \\
\hline T90 \% & $-0.174(0.184)$ & $0.025(0.861)$ & $0.298(0.157)$ \\
\hline LaSO 2 & $-0.494\left(0.000^{*}\right)$ & $-0.343\left(0.000^{*}\right)$ \\
\hline CPAP pressure & $0.443\left(0.000^{*}\right)$ & $-0.343\left(0.000^{*}\right)$ & $\square$ \\
\hline mCSA-NP & $-0.147(0.066)$ & $0.169\left(0.034^{*}\right)$ & $-0.178\left(0.025^{*}\right)$ \\
\hline mCSA-OP & $0.058(0.472)$ & $0.018(0.824)$ & $0.012(0.880)$ \\
\hline mCSA-HP & $-0.079(0.326)$ & $-0.065(0.422)$ & $0.002(0.981)$ \\
\hline UAL & $0.214\left(0.006^{*}\right)$ & $-0.181\left(0.021^{*}\right)$ & $0.348\left(0.000^{*}\right)$ \\
\hline MPH & $0.169\left(0.031^{*}\right)$ & $-0.170\left(0.030^{*}\right)$ & $0.313\left(0.000^{*}\right)$
\end{tabular}

OSA obstructive sleep apnea, CPAP continuous positive airway pressure, BMI Body mass index , AHI apneahypopnea index, ODI oxygen desaturation index, T90\% the percentage of total sleep time spent with $\mathrm{SpO}_{2}<90$ $\%, \mathrm{LaSO}_{2}$ lowest $\mathrm{O}_{2}$ saturation, mCSA-NP, mCSA-OP, mCSA-HP: The minimal cross sectional area within the nasopharynx, oropharynx and hypopharynx regions; AP/T anteroposterior/transverse axis ratio; UAL upper airway length; MPH the distance from mandibular plane to hyoid bone.

$* p<0.05$

Table 3 Stepwise multiple regression analysis of all patients using evaluate $\mathrm{AHI}, \mathrm{LaSO}_{2}$ and CPAP titration level as dependent variables

\begin{tabular}{llll}
\hline \multicolumn{1}{c}{ Dependent variable } & Independent variable & $\beta$ & $p$ values \\
\hline & BMI & 0.247 & 0.005 \\
AHI & CPAP titration level & 0.245 & $0.001^{*}$ \\
\hline$\left(\mathrm{R}^{2}=0.373, P=0.001^{*}\right)$ & Neck circumference & 0.282 & $0.001^{*}$ \\
& & & \\
\hline $\mathrm{LaSO}_{2}$ & CPAP titration level & -0.450 & $0.000^{*}$ \\
\hline$\left(\mathrm{R}^{2}=0.360, P=0.000^{*}\right)$ & Neck circumference & -0.300 & $0.000^{*}$ \\
\hline $\mathrm{CPAP}$ pressure & & & $0.000^{*}$ \\
\hline$\left(\mathrm{R}^{2}=0.222, P=000^{*}\right)$ & $\mathrm{BMI}$ & 0.343 & $0.000^{*}$ \\
\hline
\end{tabular}

OSA obstructive sleep apnea, CPAP continuous positive airway pressure, BMI Body mass index , AHI apneahypopnea index, $\mathrm{LaSO}_{2}$ lowest $\mathrm{O}_{2}$ saturation, UAL upper airway length; MPH the distance from mandibular plane to hyoid bone.

$* p<0.05$ 
Page 12/12 